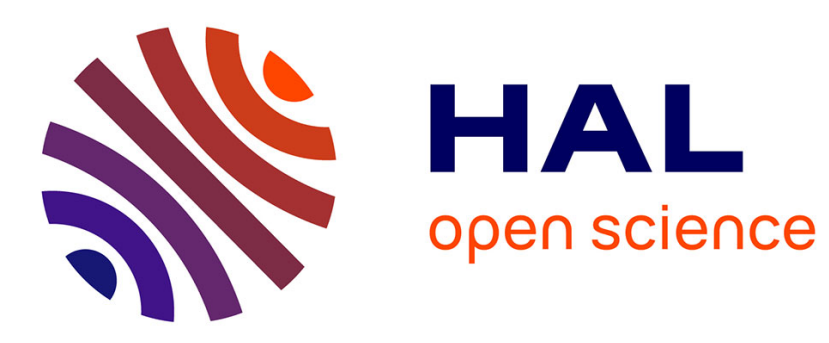

\title{
Réseau ferré, mobilités spatiales et dynamiques des territoires
}

\author{
Pierre Messulam, Nacima Baron-Yelles
}

\section{To cite this version:}

Pierre Messulam, Nacima Baron-Yelles. Réseau ferré, mobilités spatiales et dynamiques des territoires. Gérer et Comprendre. Annales des Mines, 2013, http://www.annales.org/gc/2013/resumes/septembre/01-gc-resum-FR-AN-AL-ES-septembre2013.html\#01AN. hal-00923785

\section{HAL Id: hal-00923785 https://hal.science/hal-00923785}

Submitted on 4 Jan 2014

HAL is a multi-disciplinary open access archive for the deposit and dissemination of scientific research documents, whether they are published or not. The documents may come from teaching and research institutions in France or abroad, or from public or private research centers.
L'archive ouverte pluridisciplinaire HAL, est destinée au dépôt et à la diffusion de documents scientifiques de niveau recherche, publiés ou non, émanant des établissements d'enseignement et de recherche français ou étrangers, des laboratoires publics ou privés. 


\title{
Réseau ferré, mobilités spatiales et dynamiques des territoires
}

\author{
Pierre Messulam, Directeur de la Stratégie SNCF \\ Nacima Baron, Université Paris Est
}

\section{Introduction}

Le réseau ferré français, durant plus de cent soixante années, a modelé le territoire national, offert des opportunités de développement aux territoires, aux activités économiques et aux hommes, et façonné les habitudes de mobilité de nos concitoyens et de leurs marchandises. S'agissant plus précisément du transport ferroviaire de voyageurs, qui fait l'objet du présent article, les interactions entre les tendances de répartition de la population, les besoins de mobilité et l'évolution du réseau ferroviaire constituent une question clé qui trouve son actualité dans les débats liés au Schéma National des Infrastructures de Transport.

Dans les trente dernières années, deux faits essentiels ont profondément remanié le dessin du réseau en étoile issu du $\mathrm{XIX}^{\mathrm{e}}$ siècle : la grande vitesse ferroviaire, d'une part, et la régionalisation des chemins de fer. Toutes ces évolutions ont contribué à une évolution des habitudes de déplacement des Français, à mesure que s'est constitué un régime de relations entre les lieux, du fait de l'accessibilité relative créée par le chemin de fer entre les villes, petites et grandes, entre les métropoles et leurs périphéries. Mais ce système d'accessibilité est-il aujourd'hui vraiment cohérent avec les attentes des populations et des territoires au regard des tendances dessinées par les travaux de prospective?

Les institutions de veille et de prospective soulignent qu'une nouvelle relation de la société française à l'espace est en train d'apparaître. Les manières de vivre, de travailler, de consommer évoluent et se diversifient. Loin de chercher seulement à minimiser les coûts, nos concitoyens maximisent leurs opportunités par des choix rationnels, en prenant en compte certes, les contraintes budgétaires croissantes, mais aussi les possibilités d'emploi et de résidence dans des aires élargies. Leurs comportements de mobilité (de la mobilité résidentielle à la mobilité quotidienne domicile-travail, mais aussi les mobilités de loisir) sont donc essentielles pour planifier les réseaux de demain.

Cette synthèse présente d'abord les grandes phases de l'évolution du réseau ferroviaire français et propose quelques considérations sur l'accessibilité relative des territoires, puis esquisse les tendances de re-localisation de la croissance de la demande de trafic ferroviaire voyageur à l'horizon 2040, en croisant les facteurs de mobilité de l'activité économique et de la croissance démographique. II en découle, enfin, une mise en perspective des enjeux de planification, de gestion, et de gouvernance future des réseaux ferroviaires dans un contexte d'ouverture à la concurrence. L'enjeu essentiel est de développer des outils plus performants pour hiérarchiser, avec clairvoyance, les projets de rénovation comme les nouveaux projets d'infrastructures les plus adaptés aux attentes des territoires et à l'intérêt collectif. 


\section{Réseau ferré national : un déploiement en quatre étapes}

\section{Dans années 30 , le train au service d'une fine irrigation du territoire}

La morphologie actuelle du réseau ferré français constitue essentiellement un héritage de la trame laissée par la première phase de construction au $\mathrm{XIX}^{\mathrm{e}}$ siècle et au début du $\mathrm{XX}^{\mathrm{e}}$ siècle. Elle garde de cette longue période sa structure fondamentale, c'est-à-dire le schéma radial qui organise la convergence des lignes vers la capitale. C'est dans les années 1930 que le réseau atteint son plus long développement linéaire et qu'il compte le plus grand nombre de lignes en service. La carte de 1938 reproduite ci-contre présente une trame d'une finesse géographique remarquable, avec un extraordinaire maillage, notamment dans les deux tiers nord de la France. Même si les vitesses sont relativement faibles, la fréquence des liaisons assure un fort degré de connexité et une qualité relative de relations entre les villes petites et moyennes, notamment là où le réseau est le plus serré.

Insérer Figure 1 Le réseau ferré français atteint son plus

long développement linéaire dans les années 1920.

Cette carte nous montre donc que la relation entre réseau et territoire n'est pas forcément celle d'une disjonction ou d'un divorce. D'un côté, le réseau ferré a suscité de fortes croissances urbaines, et même la création de nombreuses petites villes vouées à la villégiature (pensons à Cabourg, Arcachon, ...), ces stations étant planifiées par les compagnies privées alors concessionnaires du réseau. Le réseau ferroviaire est également créateur de relations privilégiées entre des pôles urbains, ce qui réorganise toute la géographie de la production et des échanges. Ainsi, si la ville de Saint-Etienne doit son formidable développement à l'exploitation du charbon, c'est parce qu'un canal reliant les zones d'extraction à la Loire assurait dès le début du $\mathrm{XIX}^{\mathrm{e}}$ siècle la distribution du minerai à Paris. Avec la création du réseau ferré, Saint-Etienne se détourne de l'Orléanais et nourrit la croissance industrielle lyonnaise. "Le chemin de fer est plus un révélateur qu'un facteur de discrimination urbaine", évoque la géographe Denise Pumain ${ }^{1}$.

II reste de cette longue époque un lien très puissant entre l'espace français et le train, comme il demeure chez nos concitoyens des modes de perception et d'usage de l'espace hérités et transmis par ces habitudes. Ainsi, si Quimper est si "loin" de Brest aujourd'hui dans les mentalités, c'est que les deux villes finistériennes furent desservies par deux lignes différentes depuis Paris et que cette discontinuité ne leur a pas permis d'échanger beaucoup (du moins avant les années 1970 et le plan routier breton). L'une garda donc son développement industriel et naval, l'autre sa vocation commerçante et bourgeoise. La force centripète du réseau français est telle qu'elle dépasse nos frontières et marque même les réflexes de nos voisins. L'étoile du réseau ferré français exerce un rayonnement à l'échelle européenne dès sa création ${ }^{2}$.

\section{Les années 1960-70, la première modernisation du réseau}

L'époque des "Trente Glorieuses" accorde un fort avantage à la planification du mode autoroutier et routier et promeut le déploiement des lignes aériennes intérieurs. Mais c'est aussi l'époque au cours de laquelle le réseau ferré connaît une première contraction et

1 Pumain D. "Chemin de fer et croissance urbaine au XIX ${ }^{e}$ siècle", Annales de géographie, $\mathrm{n}^{\circ}$ 507, sept octobre1982, p. 529-548 citation p. 536.

2 Germá Bel España, capital París , éditeur Destino, 2010 
restructuration sous l'effet de quatre facteurs. Le premier est l'abandon des petites lignes rurales (et en montagne) consécutivement à leur baisse de fréquentation. Le réseau atteint son stade $d$ edéploiement maximal EN \&ç"à ;EXODE RURAL Le second est le renforcement des échanges de tous types (bancaires, téléphoniques, de main d'oeuvre, ... et ferroviaires) entre les "métropoles d'équilibre" et Paris. En effet, une ambitieuse politique de rééquilibrage urbain est portée par le Délégué à l'aménagement Olivier Guichard. Dans un esprit de "géographie volontaire ${ }^{3 ",}$ des organismes spécialisés, les OREAM ${ }^{4}$ encouragent le développement des huit métropoles d'équilibre (Lyon-Grenoble, Marseille, Strasbourg, MetzNancy, Lille, Nantes-Saint Nazaire, Bordeaux, Toulouse). L'objectif est de mieux répartir sur l'ensemble du territoire les fruits de la croissance économique. L'une des meilleures preuves de la réussite de ce pari est l'élévation des niveaux de trafic entre des ensembles urbains, notamment sur les grands axes radiaux, en particulier les vallées de la Seine et du Rhône. Les réseaux se renforcent donc entre une dizaine de villes françaises et Paris. L'impact de la modernisation des matériels joue aussi : la couleur orange, typique des trains Corail introduits à partir de 1975, est synonyme à l'époque de confort (chauffage dans les trains, insonorisation), de rapidité $(200 \mathrm{~km} / \mathrm{h})$. Un troisième facteur explique la densité des relations entre les villes moyennes de la moitié nord et l'agglomération parisienne : c'est la politique de déconcentration industrielle ${ }^{5}$. Le réseau renforce dans l'orbite du grand bassin parisien, les relations de Paris, où demeurent localisés les grands sièges sociaux, avec des villes moyennes industrielles : Chartres, Angers, Orléans, Amiens, Rouen, Sens, ... Cette couronne de pôles secondaires allie fort développement démographique et économique et bonne connexion à Paris, donc à la France entière. Enfin, la création du réseau express régional (RER) en lle-de-France constitue une innovation importante qui répond à la profonde transformation administrative, industrielle, et urbaine de la région capitale. Les lignes de RER desservent les quatre villes nouvelles et les grands pôles d'affaires (La Défense) avec le centre de Paris, mais accompagnent aussi le mouvement de desserrement résidentiel de la deuxième couronne.

\section{La "révolution ferroviaire" des années 1990}

Deux événements majeurs bousculent à partir des années 1980 le fonctionnement des relations ferroviaires au sein de l'espace français, et, partant, la qualité des relations entre les villes françaises. En premier lieu, l'essor du réseau de lignes à grande vitesse réduit considérablement la distance temps de la capitale vers un petit nombre de métropoles régionales. L'attractivité du marché sud-est pousse à l'ouverture de la ligne Paris-Lyon en 1981. L'ouverture de la ligne atlantique (Bordeaux, Nantes et Rennes en 1989) accompagne l'expansion rapide des villes de l'ouest. En 1993, Lille est reliée en moins d'une heure avec Paris, mais se connecte également avec les métropoles du Bénélux et de l'Allemagne. Les lignes suivantes présentent un attrait commercial moindre (par exemple la LGV Est, mise en service en 2007) et nécessitent donc le concours financier des collectivités territoriales desservies. Le réseau à grande vitesse se développe désormais sur des relations interrégionales (cas de l'axe Lyon Strasbourg ou de Tours Bordeaux) ou intra-régionales (Lyon - Valence, Avignon - Marseille), sachant que dans les deux cas, les impacts sur les espaces transfrontaliers (notamment avec l'Espagne, la Suisse, le Belgique, l'Allemagne, l'Angleterre sont loin d'être négligeables.

La grande vitesse modifie la hiérarchie urbaine et remodèle le système de relations entre les grands pôles urbains. Ainsi, les "villes TGV", comme elles aiment à se nommer, tirent parti de l'accessibilité à la capitale pour capter de nouvelles fonctions métropolitaines (avec les grands équipements culturels : Beaubourg à Metz, Le Louvre à Lens) mais aussi pour élargir leur avant et leur arrière-pays ${ }^{6}$. Au passage, au seuil des années 1990, l'ouverture des lignes

\footnotetext{
3 Jean Labasse L'organisation de l'espace. Éléments de géographie volontaire Paris, Hermann, 1966,

${ }^{4}$ OREAM Organisme de recherche, d'étude et d'aménagement des aires métropolitaines

5 Michel Michel Chartres, Dreux, Evreux, Les problèmes du développement des villes moyennes à la périphérie de la région parisienne, thèse de doctorat, 1963.

${ }^{6}$ Cyprien Richer, Pascal Berion, "Le rôle des grandes infrastructures dans la structuration des espaces régionaux
} 
Thalys et Eurostar inscrit une bonne demi-douzaine de grandes métropoles françaises dans un nouvel espace européen de relations ferroviaires. Ainsi, Londres - Avignon, Lyon Bruxelles sont des liaisons qui offrent des temps de parcours en train très concurrentiels par rapport à l'avion, de centre-ville à centre-ville ... On doit alors souligner le rapprochement des plus grands pôles urbains entre eux (notamment ceux qui sont situés sur l'axes historique, Lille Lyon Marseille), et l'extension de l'aire d'accessibilité (comprise à partir de Paris) de la moitié sud de la France. Mais le contrecoup de l'instauration de la grande vitesse ferroviaire est le fameux "effet tunnel" qui caractérise l'espace interstitiel entre deux gares TGV. De ce fait, l'accessibilité des villes moyennes aux principales métropoles et à Paris est largement conditionnée par le fait qu'elles obtiennent ou non cette gare TGV qui les inscrira dans le réseau des gagnantes. Malgré le fait que les trains à grande vitesse puissent circuler sur le réseau conventionnel et irriguer bon nombre de localités de taille moyenne, il y a donc dès les années 1990 une prise de conscience des effets très discriminants de la grande vitesse sur la notoriété et l'image des territoires ${ }^{7}$.

Le second facteur de modification sensible du système ferroviaire, dans son fonctionnement territorial, est lié à la décentralisation. Le rôle d'autorité organisatrice des conseils régionaux pour les trafics de proximité permet, à partir des années 1990, une restructuration des réseaux régionaux et de forts investissements sur les lignes TER les plus structurantes. Dans certaines régions très urbanisées (Nord-Pas-de-Calais, Rhône-Alpes), le système de relations assure une complémentarité des villes entre elles et bénéficie autant aux métropoles (qui voit leur gare muter en véritable pôle d'échange intermodal) qu'à une série de villes secondaires bien desservies. Dans d'autres régions comme la Bretagne ou les Pays de la Loire, de gros efforts d'investissement (électrification, acquisition de matériel roulant ...) permettent de développer un tissu de relations entre des agglomérations de taille moyenne assez proches les unes des autres. En Ile-de-France, la collaboration entre Etat et région via le STIF engage de nouvelles lignes de RER (la D, la $E$, Météor) pour tenter de suivre la croissance du trafic. Dans les régions les moins densément peuplées (Poitou-Charentes, Bourgogne, Centre) et les régions montagneuses (Limousin, Auvergne), le bilan économique de certaines lignes pose les questions de la pertinence de l'offre ferroviaire et de la complémentarité des modes pour assurer la continuité et l'équité territoriale du service au public.

\section{Le fonctionnement du réseau ferroviaire français en 2012 :}

Ce survol des étapes de l'organisation du réseau ferroviaire français montre que le rail est simultanément créateur (et produit) d'un certain modèle d'organisation et de fonctionnement de notre territoire. La carte du réseau ferroviaire 2012 montre une France du nord très dépendante du noeud parisien, relativement bien maillée, opposée à une France du sud à organisation plus méridienne. Cependant, il faut considérer la qualité des relations entre les villes à plusieurs échelles : l'échelle régionale, qui permet d'articuler des pôles urbains complémentaires au sein d'une même aire de déplacements, l'échelle inter-régionale ou nationale, avec la capacité - ou non - à organiser des déplacements dans la journée sur Paris; et enfin l'échelle européenne. Si l'on observe la connexité des réseaux selon ces trois échelles, on peut reconnaître, comme le montrent les travaux de Jean Varlet $^{8}$, que les Français qui habitent et travaillent sur l'axe Lille Paris Bordeaux et sur l'axe Rouen Paris Marseille sont probablement les mieux lotis. Ils vivent dans un espace ferroviaire caractérisé par une bonne connexité aux trois échelles nationale, régionale, européenne. En revanche,

\footnotetext{
; le cas de l'arrivée du TGV dans le réseau métropolitain Rhin-Rhône", revue Belgéo, janvier 2011.

Fédération nationale des Associations d'usagers des Transports FNAUT Les perdants du TGV Les effets pervers de l'exploitation du TGV sur la desserte des villes moyennes, étude réalisée par la commission TGV de la FNAUT avec l'aide financière de la DATAR.

${ }^{8}$ Jean Varlet, Géographie des relations ferroviaires en France, 219 pages.
} 
l'analyse à l'échelle régionale montre des pôles régionaux très intégrés comme Rhône Alpes, mais d'autres (Nantes, Metz Nancy et Strasbourg) dont l'articulation avec les sousensembles régionaux est inachevée. Seules les grandes agglomérations méridionales de Nice à Bordeaux jouissent de bonnes liaisons directes entre elles, passant par Toulouse et Montpelier. Tout l'espace intermédiaire entre Nantes, Bordeaux et Lyon connaît un enclavement différentiel ou sélectif, c'est-à-dire que les connexions sur au moins deux échelles de fonctionnement sur trois sont malaisées.

insérer Figure 2 a et $2 \mathrm{~b}$

Représentation de la contraction de l'espace français par la grande vitesse ferroviaire en 1989 (2a) et 2010 (2b)

Une comparaison de deux cartes par anamorphoses du réseau entre 1989 et 2010 montre des modifications très notables du rapport espace-temps. D'un schéma à l'autre, Paris n'est plus tout à fait au centre du pays, il a glissé vers le nord, tandis que les plaines du nord de la France ont été "effacées". La hiérarchie des pôles urbains est bouleversée, Lille est plus "près" de Paris que Rouen ou Amiens, Avignon est plus proche de Paris que ClermontFerrand ou Bordeaux. La hiérarchie urbaine et la structuration des circulations entre les métropoles dans le quadrilatère Le Havre - Paris - Strasbourg - Lille sont donc réorganisées. Le raccourcissement de la distance-temps entre la France du nord et la Méditerranée est également remarquable, mais profite surtout aux villes de l'axe rhodanien et à Marseille. Cet "écrasement" des distances méridiennes contraste avec le maintien de la rugosité des distances longitudinales entre l'ouest (pointe bretonne, grand sud ouest) et le sud est, mais aussi sur la ligne de crêtes d'Annecy à Nice par Gap. Le tracé géographique des côtes atlantiques et de la frontière des Pyrénées est respecté d'une anamorphose à l'autre, ce qui montre la résistance du territoire "réel" au rapprochement métropolitain produit par la grande vitesse ferroviaire.

\section{Peuplement de l'espace français et mobilités : l'apport de la prospective}

\section{Dynamiques d'occupation du sol et réseaux : quelles interactions ?}

Les grands travaux de prospective de la fin des années 1990 coïncident avec une phase d'accélération de la restructuration institutionnelle des territoires (avec l'acte II de la décentralisation et le développement intercommunal) et avec une période d'intense mutation de l'occupation du sol. L'heure n'est plus à l'exode rural mais à la redistribution de la croissance démographique dans les périphéries des agglomérations. Le desserrement résidentiel fonctionne comme une onde qui, en fonction d'un certain nombre de facteurs (la puissance démographique du pôle émetteur, l'orientation des marchés fonciers et immobiliers ruraux, la vulnérabilité des structures foncières et agricoles, ...) développe la fonction résidentielle dans une première, puis dans une deuxième, voire dans une troisième couronne périurbaine. II est accompagné par un second facteur essentiel, la dispersion des pôles d'emploi dans les agglomérations. L'effondrement de l'emploi agricole, la stagnation du secondaire et le triomphe du tertiaire créent les conditions d'une métropolisation. L'emploi de bureau se concentre dans certains pôles centraux ou péricentraux, mais glisse aussi vers des zones d'activités péri-urbaines intégrant des fonctions commerciales.

Toutes les instituts statistiques, en premier lieu l'INSEE, sont obligés de réorganiser leurs critères de classification et de dépasser l'opposition ville / campagne (ou entre commune urbaine / commune rurale). Le premier concept opératoire forgé dans les années 1980 est celui d'agglomération, en prenant le critère de la continuité du bâti. Dans les années 1990, les premières tentatives de caractériser les unités urbaines, en fonction de l'intensité des flux d'échanges d'actifs conduit à l'invention de la zone de peuplement industriel et urbain qui 
englobe une ville centre et des communes rurales qui participent au fonctionnement de l'agglomération (par l'envoi de navetteurs). II ressort que $96 \%$ des Français vivent en ZPIU, ce qui montre que le mode de vie des Français est urbain. Finalement, une formule plus discriminante est mise au point avec la nomenclature en "aire urbaine" qui prend en compte un niveau suffisant de stocks (d'habitants et d'emplois) et de flux (notamment de déplacements domicile-travail). La limite inférieure de l'aire urbaine est fixée à 5000 emplois, celle des pôles ruraux à 2000 emplois, les autres étant des communes mono ou multipolarisées, c'est-à-dire vivant sous la dépendance des pôles d'emplois représentés. Cette nomenclature s'enrichit de la représentation d'"oursins" qui représentent l'organisation des services de proximité : un point représente une commune bien équipée, un trait relie les commune dépendantes des équipement de services à leur chef-lieu. Cette juxtaposition de l'emploi et des services permet de déterminer vraiment quels sont les territoires de la vie quotidienne : c'est ainsi que la carte dite des "territoires vécus" produite par Christophe Terrier $^{9}$ transforme la vision du fonctionnement du territoire.

Une nouvelle vision de l'articulation entre les réseaux et les territoires fonctionnels se dessine donc au début des années 2000. La carte des espaces vécus montre que la périurbanisation crée progressivement un alignement de l'urbanisation nouvelle sur les axes naturels : chapelets fluviaux, littoraux, grandes vallées ... c'est-à-dire sur les grands réseaux d'infrastructures de transport. Le géographe Paul Claval se prend à imaginer le progressif "alignement" du peuplement sur les réseaux. II formule un scenario idéal : "Les réseaux les plus efficaces sont structurés autour des grands axes et des grands pôles : cela crée une inégalité. On peut penser que la situation va devenir moins injuste parce que la population viendra s'agglomérer autour des points et des lignes les plus forts du pays. L'objectif d'équité sera réalisé en même temps que celui d'efficacité, au prix d'une inégalité dans le traitement de l'espace."

Hélas, équité, égalité et efficacité du réseau ne se répondent pas miraculeusement. Les mobilités ne "suivent" pas mécaniquement les réseaux même si ces derniers les orientent quelque peu. C'est pourquoi des travaux ultérieurs, portés par des spécialistes des mobilités, comme J.P. Orfeuil ${ }^{10}$, mettent l'accent sur l'observation sociologique des mobilités contemporaines et futures, en prenant en compte des facteurs essentiels : les vitesses, les distances, les coûts relatifs assumés par ces ménages. Toutes ces évolutions des liens entre société et territoire modifient durablement la nature, la forme et le sens des mobilités.

Insérer figure $\mathrm{n}^{\circ} 3$ figure 3

légende : La carte des territoires vécus montre la structuration de l'espace français en une myriade de centres de taille assez restreinte des pôles urbains, insérés dans des espaces résidentiels plus larges, et identifie par des traits les mobilités périurbaines (emplois, services).

source : Christophe Terrier DATAR

\section{Des valeurs sociales montantes associées à la mobilité}

Beaucoup de travaux sociologiques récents décrivent la montée en puissance de la valeur mobilité pour les Français. Cette notion s'inscrit comme une valeur structurante dans le processus de modernisation de la société française, en lien étroit avec la dynamique d'individuation : c'est l'accentuation des "capacités mobilitaires individuelles". Bien sûr, il ne

\footnotetext{
${ }^{9}$ www.christophe-terrier.com/

10 J. P. Orfeuil, Mobilités urbaines, l'âge des possibles, Ed. Scrineo, 2008
} 
faut pas entendre par là que les Français ont tous accès à une mobilité totale, ou parfaite. II y a au contraire des "inclus" qui ont les moyens et l'envie de se déplacer (à la fois dans les mobilités résidentielles, les mobilités domicile-travail, les mobilités touristiques ou de loisirs) et qui ont donc accès à un vaste répertoire de lieux, de sites, de situations de vie. De l'autre côté, il existe effectivement des "exclus" partiels ou absolus de la mobilité mais ces derniers partagent avec les premiers l'idée que la mobilité est positive, qu'elle est associée à une image de modernité, d'émancipation, d'ouverture des possibles relationnels ou professionnels.

Le second constat est que la capacité mobilitaire des sociétés modernes (ou postmodernes), si valorisée socialement, est étroitement liée avec une volonté de maîtrise, et d'organisation du temps. La contraction et l'intensification des horaires et des formes de travail, la valorisation des loisirs, mais aussi l'allongement de la vie ont transformé l'appréhension de la temporalité par les Français. La valeur croissante de la mobilité interagit avec la valeur du temps que l'on a à soi, que l'on s'accorde, que l'on négocie dans un contexte de contraintes multiples. Les Français essaient, chacun en fonction de leurs budgets, de leurs représentations, de profiter de toute l'étendue offerte par les réseaux et par les vitesses plus grandes des moyens de transport, mais aussi d'employer au mieux leur temps (quotidien, hebdomadaire, annuel ou sur la durée de la vie). II faut donc lire les choix des Français en termes de localisation résidentielle, de choix du lieu d'emploi, de rapport aux différents modes de transports plus ou moins rapides et aisés (automobile ou transports collectifs) comme autant de choix rationnels sous contraintes (contraintes budgétaires et choix de durée maximum des liaisons dans le budget temps quotidien). L'usage des technologies de communication, associées de manière de plus en plus systématique aux déplacements physiques, s'explique aussi par ce besoin de gain de temps.

\section{Les migrations au sud et à l'ouest : les emplois suivent, mais les réseaux ?}

Les travaux sur les effets de la mobilité intra-régionale (mobilité périurbaine et métropolisation) ont quelque peu passé sous silence un second phénomène, pourtant très lourd de conséquences, qui est la mobilité interrégionale. Cette seconde forme de mobilité des Français (déplacement du lieu de résidence) et, dans un second temps, déplacement des emplois, a pourtant démarré dès les années 1980.

Deux informations importantes ont causé l'étonnement des géographes au tournant des années 2000. C'est à l'occasion du recensement de 1999 que, pour la première fois depuis plus d'un siècle, la région parisienne s'est révélée déficitaire en matière de solde migratoire. Cette information était difficile, a piori, à articuler avec le constat de l'urbanisation généralisée de la France, avant qu'on ne mette en évidence que l'aire urbaine de l'agglomération parisienne en était simplement venue à "déborder" assez largement des limites régionales. Les géographes ont alors appris à identifier le fonctionnement de ces territoires de la frange francilienne qui vivent "à l'ombre" de la métropole (Oise, Loiret, Yonne...). La typologie des communes présentée dans la carte ci-contre, souligne visuellement ce vaste "halo" vert clair des communes multipolarisées de lointaine périphérie, non seulement autour du bassin parisien mais autour de Lyon, Bordeaux ou Toulouse.

En outre, deuxième étonnement des spécialistes : la zone proche des frontières allemandes et du Bénélux, cette portion de territoire qui est au contact de l'arc de prospérité unissant les 
villes européennes de l'Italie du nord au Rhin et au bassin londonien, s'est révélée beaucoup moins attractive (en termes de mobilité résidentielle) que les zones littorales atlantiques et méditerranéennes. Maurice Ulrich avait été l'un des premiers, au milieu des années 1980, à anticiper cette "revanche des suds" en soulignant les facteurs d'attractivité ${ }^{11}$ de ces territoires : les aménités naturelles bien sûr (climat, proximité des rivages ou des sommets neigeux), mais aussi les stratégies de développement des technopôles (avec Sophia Antipolis, puis les centres de recherche publics et privés transférés, comme IBM à Montpellier ou Hewlett Packard à Grenoble), et enfin les retombées de l'économie touristique. Depuis, la déconnexion des zones de production et de consommation de la richesse en France, la croissance démographique des régions du sud et de l'ouest, la spécialisation de certains territoires dans la captation de revenus de transfert (retraites, allocations sociales) deviennent de plus en plus évidents. Cela conduit l'économiste Laurent Davezies à proposer le concept d'économie résidentielle (articulée ou non avec le développement de fonctions tertiaires supérieures) pour expliquer cette dynamique très visible sur la carte ci-contre.

\section{insérer Figure 4 : La construction de logements dans l'espace français : le poids des périphéries}

La base de données SITADEL (réalisée à partir des demandes de permis de construire) dont est extraite la carte montre une importante poussée de construction résidentielle sur les littoraux et les zones frontalières du sud et de l'est de la France. source : Etude EDATER pour la DATAR

\section{L’attractivité des interstices : une mobilité libérée des réseaux ?}

II est certain que le développement des grands réseaux d'infrastructures ferroviaires comme la LGV Méd ${ }^{12}$ - a eu un effet direct et immédiat sur l'attractivité de certains territoires : pensons à l'essor de Valence (la zone d'activité et les effets sur l'emploi). Les trafics ayant pour origine ou destination la vallée du Rhône, le Languedoc-Roussillon et Provence-Alpes Côte d'Azur ont fortement crû, et sont appelés à croître encore (voir la carte ci-contre prévisions de trafic à 2020). Cette tendance montre que le réseau à grande vitesse stimule indiscutablement l'activité sur les territoires, mais aussi les fréquentations touristiques, les courts séjours et les activités de loisirs vers le sud de la France. On peut noter également qu'il a entraîné un renchérissement du prix des biens immobiliers (voués à la fonction de résidence secondaire comme à la résidence principale) pour des segments de population, soit retraités, soit actifs dits "métropolitains supérieurs".

Un autre phénomène, qui ne touche pas le sud de la France uniquement mais qui y est plus prégnant qu'ailleurs, s'est aussi accentué dernièrement. L'enquête diligentée par la DATAR autour des années 2010 a documenté avec précision ${ }^{13}$ la fuite résidentielle des zones métropolitaines vers des zones de moindre pression. A l'échelon départemental, Ardèche, Aveyron, Lozère, Ariège connaissent tous un regain démographique non négligeable, qui est en partie le fait de retraités, en partie le fait de jeunes actifs. A l'échelon encore plus fin, celui du canton ou de l'intercommunalité, il devient vraiment difficile d'identifier ce que l'on appelait dans les années 1980 des zones en déprise. Au sud de la Loire, on ne trouve quasiment plus de zone rurale qui soit véritablement en crise démographique. Ailleurs, (Dordogne, Lot, Luberon, ...) la faible densité est désormais valorisée, fréquentée selon des rythmes et des intensités variables, et intégrée au système métropolitain et aux logiques de mobilité de nos concitoyens et de nombreuses populations étrangères. Certains Français et nos voisins

\footnotetext{
${ }^{11}$ Maurice Ulrich La revanche des suds, 1985.

12 RFF SNCF Bilan LOTI de la LGV Méditerranée, 2007, 117 pages.

13 Emmanuel Roux, Martin Vanier La périurbanisation : problématiques et perspectives La Documentation française, 2008, $87 \mathrm{p}$.
} 
européens (Belges, Anglais notamment) s'y installent définitivement après une vie active. Dans des zones moins réputées (Creuse, Limousin et Charente, contreforts du massif central et des Pyrénées) s'installent des familles qui tentent un autre "projet de vie". La mauvaise accessibilité de ces arrière-pays n'est pas forcément un désavantage, elle peut même se renverser, quand un tissu minimum de services publics garantit la viabilité de ces territoires : le maintien de réseaux éducatifs (écoles de village) et les réseaux de santé rendent ou non la vie possible au quotidien. La disponibilité d'internet et des réseaux de téléphonie mobile jouent également un rôle dans le développement de ces mobilités résidentielles ou touristiques (quelquefois un peu hybrides entre ces deux catégorisations), dans la diffusion de l'habitat - et même des activités - très loin des métropoles, dans des périphéries peu denses, et dans les formes d'hybridation des modes de vie (entre rural et urbain) qui s'y inventent.

Au total, le tableau des mobilités des Français s'enrichit, se complexifie, et ne marque pas vraiment le pas. Malgré la crise. Les Français sont et resteront mobiles. Ils présentent une "appétence territoriale" forte, ils font montre d'une envie et d'une capacité à se déplacer sur l'ensemble du territoire. La "métropolisation à la française", qui induit la mise en relation des espaces les plus denses aux plus déserts, des plus urbanisés aux plus sauvages, repose aujourd'hui pour l'essentiel sur le mode automobile et, dans une bien moindre mesure que par le passé, sur le mode ferroviaire. Mais, sous l'effet du renchérissement du prix de l'énergie et de la raréfaction des hydrocarbures, le transfert progressif de ces mobilités vers des modes collectifs et plus économes en énergie est certain. Ce phénomène se produit déjà sous nos yeux, avec l'accroissement rapide des trafics TER et transiliens par exemple. Il faut donc réfléchir aux conditions d'adaptation de l'offre ferroviaire, tant en matière d'offre de réseau que de débit et de service pour répondre à la demande future.

\section{Réseau ferré et dynamiques métropolitaines : quelques défis majeurs}

Le regard rétrospectif posé sur la structure du réseau ferré français et l'analyse plus prospective relative aux comportements de mobilité conduit à proposer quelques concepts éclairant les stratégies de l'acteur ferroviaire demain. L'enjeu essentiel est de ne surtout pas considérer les infrastructures isolément du territoire mais, au contraire, de tenter de penser ces dernières en lien avec les logiques territoriales et les pratiques spatiales des Français, de manière à ce que le rail accompagne ou suscite des manières d'habiter, de travailler, de consommer plus durables.

\section{Mieux connecter les flux métropolitains}

Quelles sont ces logiques ? La représentation qu'en donne la DATAR dans l'exercice réalisé à l'horizon 2040 dans la figure 5 met l'accent sur une lecture à double échelle du fonctionnement métropolitain français. On remarque dans un premier temps la "sphère" francilienne (un "Paris" "à plus de 11 millions d'habitants), connectée par les lignes à grande vitesse construites (traits continus) ou en projet (tirets discontinus) avec une bonne douzaine d'autres entités. C'est de la qualité des liens entre nos grands pôles urbains et entre ces derniers et l'étranger que découle la compétitivité globale de notre pays. L'enjeu, du point de vue du réseau ferroviaire ne consiste plus à renforcer tel ou tel axe du réseau Paris / province pour gagner quelques minutes mais il est double : d'une part de travailler l'intermodalité sur les derniers kilomètres et d'autre part d'optimiser les interactions entre ces grands pôles, de valoriser leur complémentarité, en jouant sur leurs spécificités, en accompagnant les demandes de mobilité des actifs par une offre de trains Intercités ou TGV à des tarifs bien échelonnés, et selon des fréquences suffisantes. 


\section{insérer Figure 5}

Ce cartogramme est extraite de l'exercice de prospective France 2040 de la DATAR (K. Hurel) et représente l'organisation de l'espace français comme un système métropolitain interconnecté

Dans un deuxième temps, on perçoit à l'intérieur de chacune de ces sphères les tracés communaux des aires urbaines élargies et on prend en considération la mosaïque complexe de communes plus ou moins violettes (il faut comprendre plus ou moins riches), plus ou moins cerclées de vert (plus ou moins solidaires sur le plan intercommunal). Ce tracé souligne l'hétérogénéité des grandes métropoles, leurs polarisations sociales internes, et les risques de fragmentation. La concentration des valeurs foncières et immobilières définit au sein de ces métropoles des processus de spécialisation fonctionnelle (bureaux, logements) et de ségrégation socio-spatiale. Le second défi est donc d'assurer la viabilité de ces ensembles métropolitains à long terme en réussissant une mise en relation suffisante des lieux d'habitat et de transport. Ceci nécessite de dépasser, dans la structuration des réseaux ferroviaires (tramways, RER) des grandes villes, l'approche traditionnelle centre-périphérie et aller vers une valorisation des transversales de banlieue à banlieue et à une approche de type "mass transit".

Les villes moyennes représentent le troisième maillon du système métropolitain, mais elles ont besoin d'une accessibilité repensée et d'une meilleure qualité de service (distance-temps et distance-coûts, ponctualité et confort ...) avec la métropole régionale. La solution unique du train express régional n'est certainement pas la plus adaptée sur tout le territoire et pour toutes ces villes. C'est en fonction de la morphologie du territoire, des caractères de densité et de linéarité de l'urbanisation, de l'importance des trafics et des sources de financement disponibles qu'une liaison ferrée peut être pensée, surtout si cette liaison permet une désaturation des centres métropolitains et une spécialisation des pôles urbains. Tout ceci conduit à insister sur quelques pistes de travail pour les toutes prochaines années.

\section{Des réseaux ferroviaires conjugués avec les autres modes de transport...}

Dans les grandes aires urbaines, les perspectives de rabattement des flux automobiles domicile-travail vers les TER et les trains de banlieue à 20 ans laissent augurer, si l'on garde globalement les infrastructures et les régimes de fonctionnement cloisonnés actuels (bus, métros, trams, lignes de banlieue, lignes régionales), d'une saturation probablement insupportable. Les capacités d'optimisation de la gestion des trafics formeront une source limitée de progrès, et l'augmentation des vitesses de déplacement en zone construite (car l'option souterraine est coûteuse) n'offre que peu de gains en matière de débit. L'avenir des systèmes de transport métropolitain de masse ne sera donc pas résolu sans, d'une part, une articulation meilleure des modes de transports ferroviaires avec les autres modes en zone centrale et en première couronne, et, d'autre part, sans le rabattements des voyageurs provenant des communes plus lointaines par des modes collectifs (car, bus à haut niveau de service) sur route et autoroute, selon de grands corridors qu'on perçoit déjà dans la trame des paysages. Les modes strictement individuels ou individuels / partagés (les types de covoiturage, les véhicules, électriques ou non, à deux ou quatre roues, en libre service loués sur des durées variables) devront aussi être facilement articulables avec les modes collectifs. Cette logique d'optimisation conjointe des modes ferroviaires et routiers nécessite une vision beaucoup plus décloisonnée qu'aujourd'hui de l'exploitation et de la gestion des réseaux, une capacité à faire accepter des décisions de transfert modal au nom de la rationalité économique (le ferroviaire n'a pas sa pertinence dans les zones trop peu denses).

\section{3. ... Grâce au développement de pôles d'échange multimodaux}

Il y a sans doute beaucoup d'innovations d'ordre social et organisationnel à intégrer dans ce basculement des mobilités métropolitaines vers des modes collectifs articulant rail et route, 
c'est-à-dire train, car, autopartage. Les outils technologiques (les applications de smartphone notamment) seront à l'avenir utiles pour l'information voyageurs, pour le déploiement d'une billettique forfaitisée et multimodale, et deviendront donc une des clés du fonctionnement des pôles d'échange multimodaux. Différents travaux de recherche mesurent déjà les capacités des voyageurs à se saisir de réseaux téléphoniques et internet mobiles pour développer leur "savoir-circuler"14, pour se saisir de l'ensemble des opportunités de la ville dont la gare constitue une porte d'entrée. En effet, il est assez plausible d'imaginer que le gain de temps reposera plus qu'auparavant, non sur la vitesse propre des modes, mais sur la qualité, la fiabilité et la fluidité des correspondances entre lignes d'un même réseau, entre réseaux d'un même mode, ou entre modes. L'effort doit se focaliser sur la réduction des temps de parcours terminaux, sur la diminution des contraintes liées aux ruptures de charge, aux pénibilités des transbordements...

Cela implique d'imaginer les gares et pôles d'échange comme de vrais centres multifonctionnels. Avec le développement de commerces, les zones d'interconnexions passent déjà, autour de nous, (c'est vrai dans les plus grands centres pour le moment - il faut essayer de décliner ce mouvement vers des gares de second et de troisième niveau -), d'un lieu d'articulation technique de réseaux-supports à un lieu de concentration de services. Si on parvient à articuler les systèmes de conception, gestion, exploitation des réseaux transports collectifs selon une perspective d'interconnexion et intermodale, les grandes gares deviendront alors des lieux particulièrement attractifs et polarisateurs sur le plan des activités économiques, offrant une gamme de services (tout ce qui accompagne, précède et prolonge le déplacement), de commerces, et de lieux de réunion pour le travail nomade. Pour les acteurs économiques du transport et pour les acteurs institutionnels et politiques des aires urbaines, ces pôles d'échanges et les portions d'urbanisation qui les entourent, ainsi que les espaces publics proches, les voieries d'accès, représenteront donc outils permettant de produire de la valeur pour renforcer l'offre de transport et requalifier le système territorial métropolitain dans son ensemble.

\section{Réaffirmer la vocation continentale du réseau ferroviaire}

En rénovant ou créant des gares et des pôles d'échanges multimodaux plus efficaces, les acteurs politiques et économiques vont donc, dans les prochaines années, sélectionner les portes des territoires, et ils vont influer sur la hiérarchie et la localisation des rentes foncières au sein de l'espace métropolitain. Capter une partie, fût-ce faible, de ces gains de valeur sera sans doute nécessaire pour répondre au deuxième défi qu'exige la mutation métropolitaine, à savoir l'intégration des métropoles françaises à un ensemble de cités globales. En effet le rail, autant que l'aérien et en bonne intelligence avec lui, aide les métropoles à accueillir les flux économiques et financiers mondiaux. Même s'il reste encore des tronçons manquants, ou des connexions frontalières difficiles, qui sont dues à l'histoire nationale de la construction des réseaux dans chaque pays d'Europe, de très grands réseaux sont en voie de préfiguration à l'échelle de l'Europe et dessinent des sousensembles territoriaux plus ou moins cohésifs.

\section{Conclusion}

Ces considérations débouchent sur des pistes de travail qui paraissent prometteuses. Oui, le réseau ferré français a représenté depuis sa création et continuera, à l'avenir, à être un outil et un enjeu majeur d'aménagement du territoire, en même temps qu'un levier de croissance pour les entreprises et un instrument formidable de cohésion de la société française. Le rail contribue puissamment à l'organisation des territoires, il possède des propriétés structurantes et polarisantes, il accompagne, oriente, souligne des dynamiques démographiques et explique en partie des contrastes dans la répartition des richesses.

Mais comme toute infrastructure lourde, le réseau ferré actuel comporte un effet "retard". Alors que les zones de population dense évoluent sensiblement, le réseau ferroviaire est

\footnotetext{
${ }^{14}$ Recherche Orange Lab, Laboratoire Ville Mobilité Transports
} 
victime d'un effet de " traînée » et reflète encore la distribution spatiale du peuplement d'il y a 30 à 50 ans. C'est un énorme défi que de revisiter les grands programmes d'aménagement de lignes ferroviaires en fonction du contexte et des besoins de demain plutôt qu'en fonction des demandes actuelles.

La juste appréciation de la dynamique des territoires et la prise en compte de l'intensité de la demande future doivent donc compter dans les critères de hiérarchisation des projets du schéma national. Ces éléments aideront à définir la création de valeur effective produite par de nouvelles lignes, et serviront à équilibrer le montage du financement entre les territoires bénéficiaires. En effet, au regard de l'ampleur de la dette du système ferroviaire français, on peut considérer que le Schéma National des Infrastructures de Transports (SNIT) définit le champ des possibles, pas celui le domaine du "faisable". II faudra choisir entre continuer le programme grande vitesse, mieux entretenir le réseau existant ou rattraper le retard, notamment celui pris en Ile-de-France. II semble incontestable qu'on ne pourra pas mener les trois de front, sous peine d'une dérive de la dette difficilement maîtrisable. II faudra donc se fixer des priorités. Ces choix appartiennent à la sphère politique.

Les grandes infrastructures de transport ferroviaire ne peuvent constituer la réponse à tous les besoins de transport public sur tous les territoires et pour tous les besoins de transport. Nos travaux ont en effet démontré que la demande est évolutive en fonction de la géographie et de la dynamique démographique et économique des territoires. Une des préoccupations principales doit donc être de mieux organiser l'inter-modalité pour mieux servir tous les territoires en dépensant au mieux les deniers publics.

Poour améliorer la performance du système ferroviaire, trois composantes doivent être mobilisée : l'amélioration de la fiabilité, qui nécessite d'enrayer le vieillissement du réseau et donc de poursuivre voire amplifier l'effort de régénération; les gains de capacité et de robustesse, qui nécessitent de traiter les principaux nœuds et segments saturés du réseau et de favoriser les correspondances; la vitesse, qui suppose d'achever le réseau LGV structurant mais aussi de traiter le dernier kilomètre d'accès au centre-ville. Par le passé, les exercices de programmation ont privilégié la composante vitesse. La dynamique de la demande pour les transports du quotidien et le besoin de fiabiliser leur exploitation imposent aujourd'hui un rééquilibrage. Augmenter le potentiel de l'existant, en particulier pour le mass transit dans les zones métropolitaines, et préserver en qualité le réseau, notamment par des aménagements ciblés, doivent devenir les priorités d'investissements des années à venir en matière ferroviaire.

En définitive, le système ferroviaire doit s'adapter aux réalités de la société française de demain, renforcer ses capacités là où la demande va continuer à croitre et se conjuguer encore d'avantage avec les autres transports là où il ne peut offrir à lui seul une offre pertinente.

\section{Auteurs :}

Pierre Messulam est à la tête de la Direction de la stratégie, de l'innovation, de la recherche et de la régulation à la SNCF

Nacima Baron est professeur à l'Université Paris Est, membre du laboratoire Ville Mobilité Transports. 